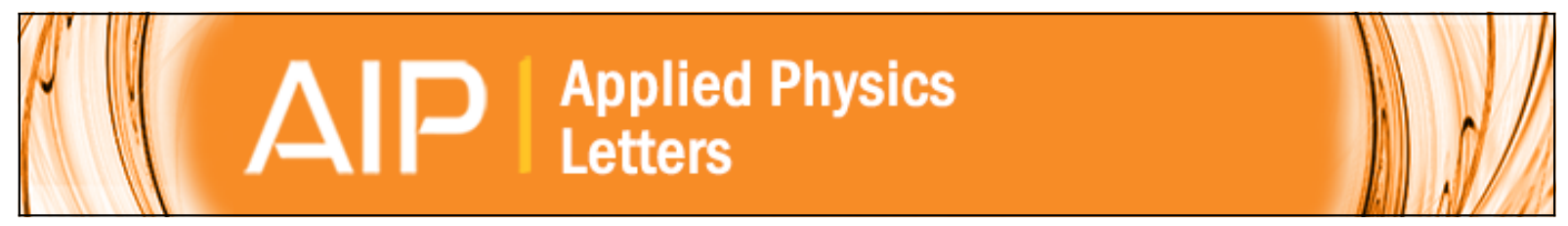

Self-consistent coupling between driven electron tunneling and electromagnetic propagation at terahertz frequencies

X. Oriols, F. Boano, and A. Alarcón

Citation: Applied Physics Letters 92, 222107 (2008); doi: 10.1063/1.2937307

View online: http://dx.doi.org/10.1063/1.2937307

View Table of Contents: http://scitation.aip.org/content/aip/journal/apl/92/22?ver=pdfcov

Published by the AIP Publishing 


\title{
Self-consistent coupling between driven electron tunneling and electromagnetic propagation at terahertz frequencies
}

\author{
X. Oriols, ${ }^{a)}$ F. Boano, and A. Alarcón \\ Departament d'Enginyeria Electrònica, Universitat Autònoma de Barcelona, 08193 Bellaterra, Spain
}

(Received 13 November 2007; accepted 6 May 2008; published online 3 June 2008)

\begin{abstract}
An accurate procedure for coupling (time-dependent) driven electron tunneling and electromagnetic propagation at terahertz frequencies cannot be developed neither with equivalent electric-circuit approximations nor using standard electromagnetic solvers. Alternatively, in this work, a full-physical time-dependent self-consistent algorithm for such coupling is presented. In order to demonstrate the numerical viability of the algorithm and to show the great interest of driven electron tunneling devices for terahertz applications, a transistorlike tunneling device (coupled to lossy transmission lines) is designed for developing a rectifier, a harmonic generator, and an amplitude modulator at terahertz frequencies. (C) 2008 American Institute of Physics.
\end{abstract}

[DOI: $10.1063 / 1.2937307]$

The partition of an experimental setup into the active region and the rest of the system, which is implicit in the analysis of most electron devices, is a delicate issue at terahertz frequencies. Since the active region interchanges (electromagnetic) energy and electrons with the micrometric wires, Maxwell and electron transport equations have to be solved self-consistently. Standard electromagnetic simulators developed for photonic systems are not valid because of their simplistic expressions for electron conductance/charge. In fact, the standard self-consistent simulation of terahertz (or millimeter-wave) electron devices follows a two-step procedure: (i) the active region and the transmission lines are "approximated" by equivalent electric circuits and then (ii) the self-consistent solution of the whole system is obtained using standard electric-circuit simulators. ${ }^{1}$ However, this procedure is not valid for driven electron tunneling at terahertz frequencies because such quantum transport regime (with scenarios oscillating at frequencies comparables to the inverse of the electron transit time) cannot be accurately described by an equivalent electric circuit (for the large signal applications discussed here). The current of driven tunneling systems can be highly nonlinear (multiple sharp resonances) and it has spatial and temporal wavelike interferences leading to complex quantum phenomena. ${ }^{2,3}$ Therefore, in this work, we present an alternative computational algorithm for the selfconsistent coupling between driven electron tunneling (in a nanoscale device) and electromagnetic propagation (in a transmission line) at terahertz frequencies. Our numerical approach is applied to a transistorlike tunneling electron device that we named driven tunneling device ${ }^{4}$ (DTD), connected through lossy transmission lines to a input voltage and a load resistance (Fig. 1). Our examples clearly demonstrate the numerical viability of our physical coupling algorithm. In addition, they also show that DTDs are interesting systems for generating/manipulating signals at the terahertz gap. Among other solid-state proposals, ${ }^{5-8}$ the DTD can be an alternative to the dominant (frequency down-conversion) optical prototypes and it can provide future terahertz systems with reduced costs, sizes, and complexities (benefitting from the state-of-the-art nanoelectronic technology).
In this work, we consider transversal electromagnetic (TEM) modes as the propagating signal along the transmission lines (wires) of Fig. 1(a), which are formulated in terms of two scalars: the current and voltage (other electromagnetic modes are also possible). At each position $x=n \Delta x$ of the wire and each particular time $t=m \Delta t$ [see Fig. 1(b)], one can define a line integral of the electric field along the distance between the two conductors to define the voltage $V(n, m)$. In addition, one can calculate the integral of the total current density on the surface surrounding the inner conductor to obtain (by Ampere's law) the total current flowing through it, $I(n, m)$. It is well known ${ }^{9}$ that the relation between the voltages and the currents in each section of the wire (transmission line) of Fig. 1(b) can be written as

$$
\begin{aligned}
& {\left[1+\Delta t \frac{G(n)}{C(n)}\right] V(n, m+1)-\frac{\Delta t}{C(n)} I(n-1, m+1)} \\
& +\frac{\Delta t}{C(n)} I(n, m+1)=V(n, m), \\
& {\left[1+\Delta t \frac{R(n)}{L(n)}\right] I(n, m+1)-\frac{\Delta t}{L(n)} V(n, m+1)} \\
& +\frac{\Delta t}{L(n)} V(n+1, m+1)=I(n, m),
\end{aligned}
$$

where $L(n)=L_{P} \Delta x$, with $L_{p}=Z \sqrt{\mu \varepsilon}$, is the inductance per unit length, and $C(n)=C_{P} \Delta x$, with $C_{p}=\sqrt{\mu \varepsilon} / Z$, is the capacitance per unit length, for a particular value of the impedance of the wire $Z$ with $c=1 / \sqrt{\mu \varepsilon}$ the electromagnetic propagation speed. On the other hand, a nonzero conductivity in the dielectric can be modeled by introducing the conductance $G(n)=G_{P} \Delta x$. In addition, the finite conductivity of the inner conductor, that affects the behavior of the waveguides and attenuates the transmitted power (i.e., skin effect ${ }^{9}$ ), can be modeled by adding the resistance $R(n)=R_{P} \Delta x$. Expressions (1a) and (1b) can we written in a matrix notation, $[M] \cdot[U]$ $=[K]$, where $[K]=\{V(n, m), I(n, m) ; \forall n\}$ is the vector of the known voltages and currents along the wire at time $t=m \Delta t$, $[U]=\{V(n, m+1), I(n, m+1) ; \forall n\}$ the unknown vector of voltages and currents at a later time $t=(m+1) \Delta t$, and $[M]$ 


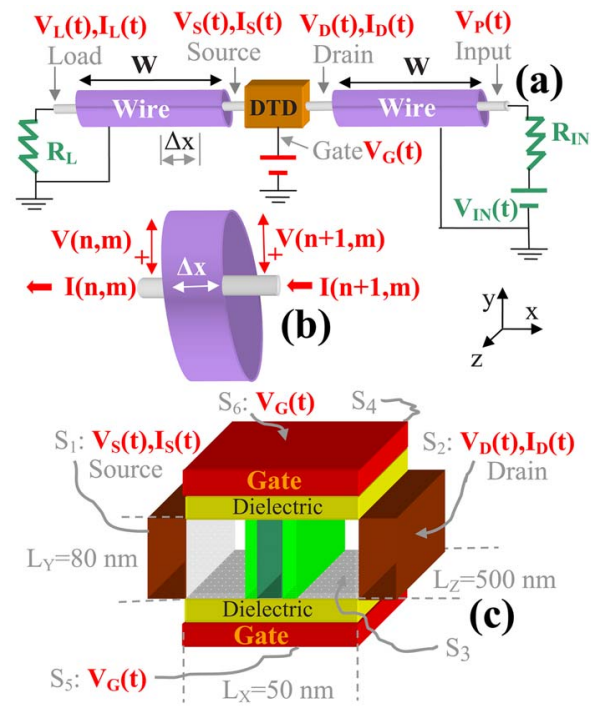

FIG. 1. (Color online) Schematic representation of the whole system (a) and of the section $x=n \Delta x$ of the lossy wire (b) with voltage and current defined at the extremes of this section at time $t=m \Delta t$. (c) $3 \mathrm{D}$ representation of the active region of the DTD and its barrier structure.

contains the fixed parameters in expressions (1a) and (1b). Simpler linear expressions are obtained for the load resistance $V_{L}(t) \equiv V(l, m)=I(l, m) R_{L}$ and the voltage input $V_{P}(t)$ $\equiv V(p, m)=V_{\text {in }}(t)-I(l, m) R_{\text {in }}$.

Next, we compute the time-dependent quantum (particle plus displacement) current through the DTD $I_{S}(t) \equiv I(s, m)$ as a function of the voltage at source $V_{S}(t) \equiv V(s, m)$ and drain $V_{D}(t) \equiv V(d, m)$ contacts. In Fig. 1(c), we consider a doublegate field-effect transistor that provides a good electrostatic gate control over the source current. A double barrier of lengths $\mathrm{dB} / \mathrm{dW} / \mathrm{dB}$ is introduced inside. The offset of the conduction band ${ }^{10}$ (above the source/drain value) are $\mathrm{oB} / \mathrm{oW} / \mathrm{oB}$. The drain and source contacts are highly doped (a Fermi level Ef far above the source/drain conduction band) to achieve a conductorlike (rather than a dielectriclike) behavior. The total time-dependent current through the source surface can be computed as ${ }^{2,4}$

$$
I_{S}(t)=I(s, t)=\Gamma^{e}(t)+\Gamma^{q}(t) .
$$

The first term $\Gamma^{e}(t)$ quantifies the dielectriclike behavior of the DTD and it contains the displacement current crossing the source surface $S_{1}$ due to the time-dependent voltage $V_{f}(t)$ on the $f=1, \ldots, 6$ surface of Fig. 1(c). It can be approximately computed as ${ }^{2}$

$$
\begin{aligned}
& \Gamma^{e}(t) \approx \sum_{f=1}^{6} C^{f} \frac{d V_{f}(t)}{d t}, \\
& C^{f} \equiv \int_{S_{f}} \varepsilon \times \vec{F}(\vec{r}) \times d \vec{s}_{f} .
\end{aligned}
$$

The $\vec{F}(\vec{r})$ can be interpreted as the electric field inside the active region (limited by the surfaces $S_{1}, S_{2}, \ldots, S_{6}$ ) without electron charge. (See Refs. 2 and 4 for the practical computation of $C^{f}$.) On the other hand, the second term $\Gamma^{q}(t)$ of expression (2) quantifies the conductorlike behavior and it contains the time-dependent quantum particle current that traverses the source surface. It can be computed as the net result of electrons injected from source and drain 2,4

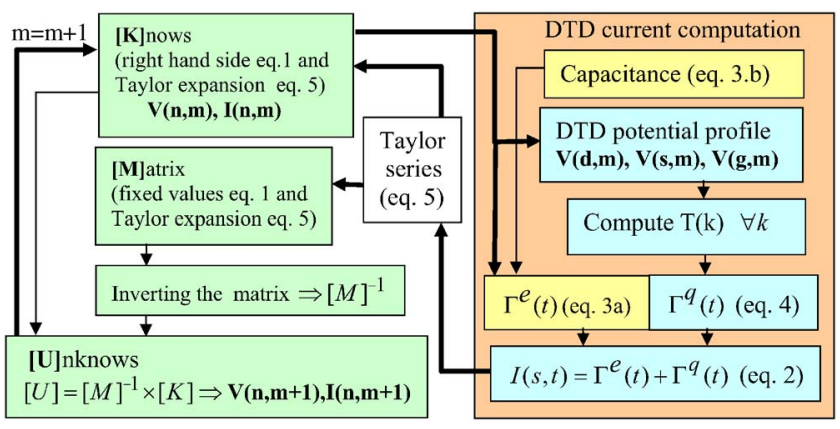

FIG. 2. (Color online) Schematic representation of the global self-consistent procedure. The right hand side explains the particular procedure for the particle and displacement current computation through the DTD at terahertz frequencies.

$$
\begin{aligned}
\Gamma^{q}(t)=\Gamma^{q S}(t)+\Gamma^{q D}(t), & \\
\Gamma^{q S / D}(t) \approx & S \frac{1}{2 \pi^{2}} \frac{q}{\hbar} k_{B} T \int_{0}^{\infty} d k_{x}\left|k_{x}\right| D^{S / D}\left(k_{x}, t\right) \ln \left\{1+\exp \left[\left(E_{f}\right.\right.\right. \\
& \left.\left.\left.-E_{x}\right) /\left(k_{B} T\right)\right]\right\},
\end{aligned}
$$

where $S=0.04 \mu \mathrm{m}^{2}$ is the source surface, $k_{B} T=0.026 \mathrm{eV}$, and $q$ is the electric charge with negative sign. The wave vector $k_{x}$ is related to the $x$ component of the electron energy (above the bottom of each conduction band) via $E_{x}$ $=\hbar^{2} k_{x}^{2} / 2 m$. We use $D^{S}\left(k_{x}, t\right) \approx \int_{-\infty}^{+\infty} d k\left|a^{j}\left(k_{x}\right)\right|^{2} T\left(k_{x}\right)$ and $D^{D}\left(k_{x}, t\right) \approx-D^{S}\left(k_{x}, t\right)$ for the computation of the current coefficients with $\left|a^{j}\left(k_{x}\right)\right|^{2}$ the energy probability distribution of the time-dependent wave-packet. At each time step $t=m \Delta t$, after computing the time-dependent potential energy profile, ${ }^{11}$ we have to solve the time-independent Schrödinger equation to find the transmission coefficient $T\left(k_{x}\right)$ of all $k_{x}$ eigenstate.

The definitions of the current at the extreme of the wires do exactly coincide with the total (particle plus displacement) current crossing the DTD. The same equivalence is achieved for the voltages at the extremes of the wires and the DTD. In order to obtain self-consistent solution of currents and voltages along the whole system of Fig. 1(a), we have to incorporate Eq. (2) into the matrix equation $[M] \times[U]=[K]$. Therefore, at each time step, the total current through the DTD $I_{S}(t+\Delta t) \equiv I(s, m+1)$ is fitted into a first-order (linear) Taylor expression of the unknown voltages $V_{S}(t+\Delta t)$ $\equiv V(s, m+1)$ and $V_{D}(t+\Delta t) \equiv V(d, m+1)$ as

$$
\begin{aligned}
I(s, m+1)= & I(s, m)+\sum_{i=S, D, G} \frac{\partial I(s, m)}{\partial V(i, m)}[V(i, m+1) \\
& -V(i, m)] .
\end{aligned}
$$

The gate voltage $V_{G}(t) \equiv V(g, m)$ is not an unknown but an input signal. For simplicity, we have assumed $I(s, m+1)$ $=I(d, m+1)$. Thus, only three derivatives have to be obtained at each time step. The voltage derivative is computed from the variation of the current when the corresponding voltage is slightly modified $(\Delta V=0.01 \mathrm{~V})$, while other voltages remain constant. Once the Taylor series is evaluated, the parameters of expression (5) can be incorporated into the definition of $[M]$ and $[K]$. We use a very small time step $\Delta t$ $=10 \mathrm{fs}$ to assure the linearity during each time step. See a complete scheme of the self-consistent algorithm in Fig. 2. The approach has been tested with analytical results for 

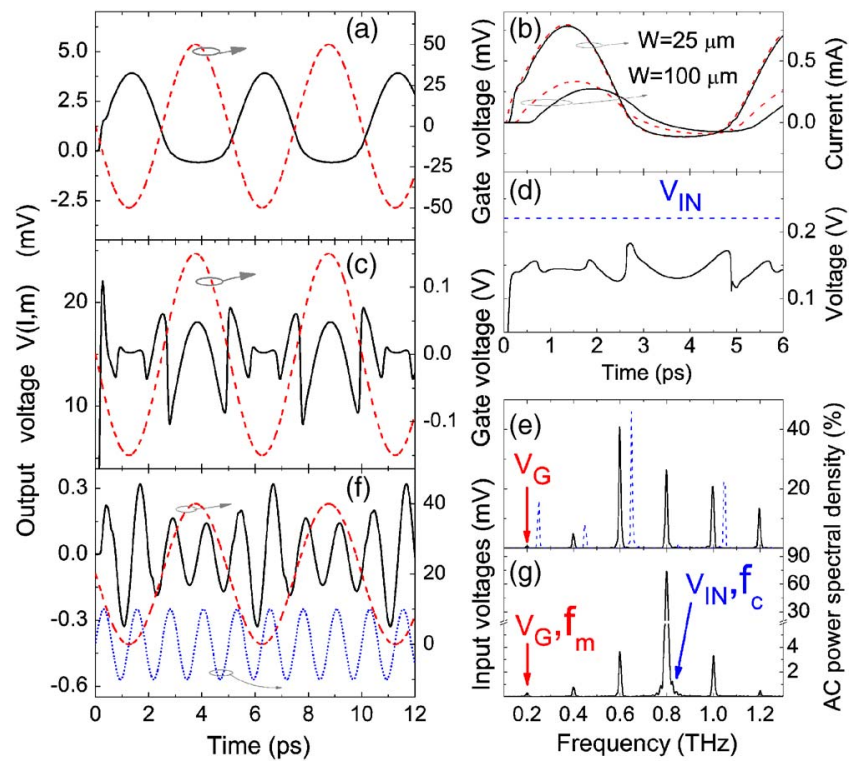

FIG. 3. (Color online) Terahertz rectifier (a) and the role of its wire length $W$ on the load current (solid line) and the DTD current (dashed line) (b). Terahertz harmonic generation (c), nonconstant voltage drop at the DTD (solid line) (d), and its power spectral density at load resistance using lossy (solid line) and lossless (dashed line with $50 \mathrm{GHz}$ shifted frequency axis, for clarity) wires (e). Amplitude modulated load current (solid line), carrier input voltage (dotted line), and modulating gate voltage (dashed line) as a function of time $(f)$ and its power spectral density at load resistance $(g)$.

single transmission lines (without the DTD) with perfect agreement.

Finally, in order to show the numerical viability of our approach and the great interest of the DTD at the terahertz gap, we develop three different terahertz applications: a rectifier, a harmonic generator, and an amplitude modulator.

In Fig. 3(a), we show a terahertz rectifier. We use a barrier heterostructure with lengths $\mathrm{dA}=2 \mathrm{~nm}$ and $\mathrm{dB}=5 \mathrm{~nm}$, and offsets $\mathrm{oB}=0.3 \mathrm{eV}$ and $\mathrm{oW}=-0.45 \mathrm{eV}$. The wire length is $W=25 \mu \mathrm{m}$ which is adapted to $Z=20 \Omega$. We use $R(n)$ $=1 \Omega, G(n)=0.04 \mathrm{mS}$, and $R_{L}=R_{\mathrm{in}}=5 \Omega$. The input gate voltage is $V_{G}(t)=0.05 \sin (2 \pi f t) \quad V$ with $f=200 \mathrm{GHz}$ [see dashed line in Fig. 3(a)]. The output voltage at the load resistance rectifies the signal [see solid line in Fig. 3(a)] because negative gate voltages produce a very opaque barrier. The role of the wire length $W$ in the current through the DTD $I(d, m)=I(s, m)$ and through the load resistance $I(l, m)$ is plotted in Fig. 3(b).

In Fig. 3(c), we show a terahertz harmonic generator. We use $\mathrm{dA}=2 \mathrm{~nm}, \mathrm{~dB}=12 \mathrm{~nm}, \mathrm{oB}=0.3 \mathrm{eV}$, and $\mathrm{oW}=-0.15 \mathrm{eV}$ that accommodate three resonant energies inside the quantum well. The wire length is $W=25 \mu \mathrm{m}$ adapted to $Z$ $=20 \Omega$ with $R_{L}=R_{\text {in }}=20 \Omega$. The input gate voltage is $V_{G}(t)$ $=0.15 \sin (2 \pi f t) V$ with $f=200 \mathrm{GHz}$ [see dashed line in Fig. $3(\mathrm{c})]$. The voltage drop along the load resistance [solid line in Fig. 3(c)] oscillates several times during a period of the input signal. The frequency multiplication is due to the fact that the DTD current acquires a maximum each time that a resonant energy of the quantum well crosses the Fermi energy. The voltage drop at the DTD $V(d, m)-V(s, m)$ [solid line in Fig. 3(d)] confirms the inadequacy of assuming a constant voltage at the wire at these frequencies, even when the input voltage is constant $V_{\text {in }}(t)=0.22 \mathrm{~V}$ [dotted line in Fig. 3(d)]. The power spectral density for output voltage using a lossy wire $[R(n)=1 \Omega$ and $G(n)=0.04 \mathrm{mS}]$ is plotted in the solid line of Fig. 3(e) to show the harmonic generation. The same data are plotted in dashed line for a lossless wire $[R(n)=0 \Omega$ and $G(n)=0 \mathrm{~S}]$ showing the important role of wire losses in the harmonic power distribution.

In Fig. 3(f), we show an amplitude modulator for terahertz frequencies. We use $\mathrm{dA}=2 \mathrm{~nm}, \mathrm{~dB}=5 \mathrm{~nm}, \mathrm{oB}$ $=0.3 \mathrm{eV}$, and oW $=-0.45 \mathrm{eV}$. The wire length is $W=25 \mu \mathrm{m}$ adapted to $Z=50 \Omega$ with $R_{L}=R_{\text {in }}=5 \Omega, R(n)=1 \Omega$, and $G(n)=0.04 \mathrm{mS}$. The gate voltage is $V_{G}(t)=0.02$ $+0.02 \sin \left(2 \pi f_{m} t\right) V$ with $f_{m}=200 \mathrm{GHz}$ and the input voltage $V_{\text {in }}(t)=0.01 \sin \left(2 \pi f_{c} t\right) V$ with $f_{c}=800 \mathrm{GHz}$ [see dashed and dotted lines, respectively, in Fig. 3(e)]. The voltage drop along the load resistance $V(p, m)$ [solid line in Fig. 3(e)] and its power spectral density [Fig. 3(f)] clearly shows a (doubleside band with carrier frequency) amplitude modulator. Interestingly, the DTD provides (the simplest) single-device modulator.

In conclusion, a self-consistent time-dependent algorithm for the difficult coupling between driven (timedependent) electron tunneling in nanoscale devices and electromagnetic TEM propagation at terahertz frequencies (without an equivalent circuit approximation) is presented. Within this full-physical self-consistent simulation of the whole (DTD plus transmission line) system, the practical ability of tunneling systems (oscillating at frequencies comparable to the inverse of the electron transit time) to manipulate/generate the terahertz signals (reducing the cost, size, and complexity of the present terahertz prototypes) is demonstrated. Future work will consider the computation of the displacement and particle terahertz currents inside the DTD region self-consistently, at each time step, with a timedependent Poisson equation. ${ }^{11}$

This work was partially supported by the Dirección General de Investigación and FEDER through Project No. TEC2006-13731-C02-02/MIC.

${ }^{1}$ R. Lake and J. Yang, IEEE Trans. Electron Devices 50, 785 (2003).

${ }^{2}$ X. Oriols, A. Alarcón, and E. Fernàndez-Díaz, Phys. Rev. B 71, 245322 (2005).

${ }^{3}$ M. Moskalets and M. Buttiker, Phys. Rev. B 66, 205320 (2002).

${ }^{4}$ X. Oriols, A. Alarcón, and L. Baella, Solid-State Electron. 51, 1287 (2007).

${ }^{5}$ N. Orihashi, S. Suzuki, and M. Asada, Appl. Phys. Lett. 87, 233501 (2005).

${ }^{6}$ W. Knap, J. Lusakowski, T. Parenty, S. Bollaert, A. Cappy, V. V. Popov, and M. S. Shur, Appl. Phys. Lett. 84, 2331 (2004).

${ }^{7}$ W. Hafez and M. Feng, Appl. Phys. Lett. 86, 152101 (2005).

${ }^{8}$ P. Plotka, J. Nishizawa, T. Kurabayashi, and H. Makabe, IEEE Trans. Electron Devices 50, 867 (2003).

${ }^{9}$ M. Javid and P. M. Brown, Field Analysis and Electromagnetics (McGraw-Hill, New York, 1963), Chap. 12, pp. 317-373.

${ }^{10} \mathrm{We}$ assume $\mathrm{Al}_{x} \mathrm{Ga}_{1-x} \mathrm{As} / \mathrm{In}_{y} \mathrm{Ga}_{1-y} \mathrm{As}$, where $x$ and $y$ controls $o B$ and $o W$, and $m=0.067 m_{0}$ where $m_{0}$ is the free electron mass.

${ }^{11}$ The particle and displacement currents are computed from a threedimensional (3D) solution of the Laplace equation applied in the volume of Fig. 1(c) at each time step. A single-particle Schrödinger equation with a one-dimensional oscillating potential is obtained by averaging over the $y-z$ plane. The exact (3D Poisson equation) computation can be achieved using a quantum many-particle algorithm [X. Oriols, Phys. Rev. Lett. 98, 066803 (2007)], which demands a large computational time. 